\title{
Neutron/gamma discrimination enhancement: plastic scintillators high dose irradiation and recovery time
}

Eva Montbarbon ${ }^{1,2,3,}{ }^{*}$, Matthieu Hamel ${ }^{1}$, Dominique Tromson ${ }^{1}$, Marie-Noëlle Amiot ${ }^{1}$, Camille Frangville ${ }^{1}$, Romuald Woo ${ }^{1}$, Robert B. Pansu ${ }^{2}$

${ }^{1}$ CEA, LIST, Laboratoire Capteurs et Architectures Electroniques, CEA Saclay, 91191 Gif-sur-Yvette Cedex, France

${ }^{2}$ ENS Cachan, 61 avenue du président Wilson, 94230 Cachan, France

${ }^{3}$ Ecole doctorale INTERFACES, Paris-Saclay University, 91400 Saclay, France

In the context of high-energy physics experiments, particle accelerators create a significant dose of radiations, up to $10^{6}$ Gy for example at the Large Hadron Collider (LHC, Cern). Control detectors, such as scintillators, are affected by these high irradiation levels. The literature mentions that the light yield of organic scintillators dramatically drops when strongly irradiated. However, the tested scintillators can recover some of their light output with time. To the best of our knowledge, only the luminescent properties of plastic scintillators were analyzed under high radiation fluxes. But, plastic scintillators are also able to discriminate fast neutrons from gamma rays. In this work, we characterized neutron/gamma discriminating as well as emissive properties of plastic scintillators after high dose irradiations.

Two identical lab-made plastic scintillators containing a polystyrene-based matrix and two fluorophores were analyzed before and after high dose irradiation. Irradiation was performed using a Gamma-Cell 220 Excel with twelve ${ }^{60} \mathrm{Co}$ sources and a $53.7 \mathrm{~Gy} / \mathrm{min}$ dose rate at isocenter. These two scintillators were irradiated to reach a cumulative dose equal to $10^{4} \mathrm{~Gy}$.

In order to measure their neutron/gamma discrimination ability, scintillators were coupled to a Hamamatsu R7724-100 photomultiplier tube and placed in front of a ${ }^{252} \mathrm{Cf}$ source (Activity $580 \mathrm{kBq}$ ). The anode signal fed a digitizer. Scintillation pulses were then recorded and post-processed. A charge-comparison method was implemented and FoM was evaluated. Offline treatment allowed the estimation of fast and slow decay times of neutron pulses, as well as their relative intensities. In parallel, characterization of radioluminescence properties of irradiated and non-irradiated samples was performed.

Results indicate a strong modification in the neutron/gamma discrimination capability before and after high dose irradiations and a redshift of the radioluminescence spectrum. In fact, a $10^{3}$ Gy irradiation increases the FoM of both sensors by a factor 2.5 at least. Further, as recovery time passes after a $10^{4}$ Gy cumulative dose, FoM improves for scintillators and is multiplied by 6 compared to the value at the zero dose. Thanks to evaluated decay times and relative intensities, we could infer that a strong irradiation does not produce a higher yield of triplet excited states but it impacts the slow decay time of the tested scintillator. This leads to a better neutron/gamma discrimination than at the zero dose. These observations lead us to believe that the intrinsic nature of the plastic material is modified under high dose irradiation.

In this paper we present the current iteration of this ongoing work. The neutron/gamma discrimination properties are presented for both plastic scintillators and first characterization results are discussed. We show that material modification under high dose irradiation manages to an improvement in neutron/gamma discrimination opening the field to applications of very high dose measurements. 
All observations we received thanks to these strong irradiation experiments will be detailed at the conference. The experiments planned to highlight the intrinsic modifications of scintillators will also be described. 